\title{
Producción científica en comunicación en las universidades de la Comunidad de Madrid: la fuerza de la tradición
}

\author{
Ignacio BERGILLOS \\ Profesor titular, Departamento de Ciencias de la Comunicación, \\ Centre d'Ensenyament Superior Alberta Giménez - Universitat de les Illes Balears \\ ibergillos@cesag.org \\ David FERNÁNDEZ-QUIJADA \\ Profesor ayudante doctor, Departament de Comunicació Audiovisual i Publicitat, \\ Universitat Autònoma de Barcelona \\ david.fernandez@uab.es
}

Recibido: $18 / 05 / 2012$

Aceptado: 13/06/2012

\section{RESUMEN}

Este artículo tiene como objetivo analizar las dinámicas de producción científica en ciencias de la comunicación de las universidades de la Comunidad de Madrid entre 2007 y 2010 . A partir de un análisis bibliométrico de las principales revistas de comunicación, seleccionadas mediante una triangulación de bases de datos bibliográficas, se determina la producción científica de cada institución, la distribución de esta producción por revistas y el volumen y el tipo de colaboración observable. Los resultados muestran que la Universidad Complutense de Madrid es una institución de importancia central, ya que genera casi la mitad de la producción y es el principal nodo de una red de colaboraciones muy centrada en las instituciones de la propia comunidad autónoma. El peso de las universidades privadas es aún reducido y sólo destaca la Universidad San Pablo CEU, que junto a la Complutense, la Universidad Rey Juan Carlos y la Universidad Carlos III forma el cuarteto de centros más relevantes en la investigación de comunicación de la Comunidad de Madrid. Se observa también una excesiva concentración de esta producción en revistas de la propia Comunidad de Madrid e incluso editadas por la propia universidad en algunos casos, así como pautas de colaboración específicas para cada centro.

Palabras clave: producción científica, revistas científicas, bibliometría, estudios de comunicación, Madrid

\section{Scientific production in communication at the Community of Madrid universi- ties: the strength of tradition}

\begin{abstract}
This article aims to analyze the dynamics of scientific production in communication sciences in universities from the Community of Madrid from 2007 to 2010. Based on a bibliometric analysis of the major Spanish communication journals, selected through a triangulation of bibliographic databases, this paper determines the scientific output of each institution, the distribution of this production by journals and the volume and type of collaboration identified. The results show that Universidad Complutense is an institution of central importance, since it generates almost half of the production and is the main node of a network of collaboration that is very focused on the institutions of the region. Only three other universities stand out: Universidad Rey Juan Carlos, Universidad Carlos III and Universidad San Pablo CEU -
\end{abstract}


the only significant private institution. An excessive concentration of production in journals published in the region is observed; this circumstance is even more acute in cases where articles are published in journals edited by the same institutions that produce the original research. Finally specific collaboration patters were found for each university.

Keywords: scientific production, scientific journals, bibliometrics, communication studies, Madrid

\section{INTRODUCCIÓN}

A lo largo del último lustro, el proceso de maduración de las ciencias de la comunicación en España ha traído consigo una creciente preocupación por el volumen y la calidad de la producción científica en el ámbito. De ahí han surgido trabajos que se han interrogado por los patrones de publicación en revistas científicas (Castillo, Carretón, 2010; Fernández-Quijada, 2010, 2011a, 2011b; López-Rabadán, VicenteMariño, 2011; Masip, 2011a, 2011b), la calidad de revistas y editoriales (GiménezToledo, 2011; Giménez-Toledo, Alcain Partearroyo, 2006; Giménez-Toledo, TejadaArtigas, 2012; Jones, 2007) y cierta prospectiva (Baladrón-Pazos, Correyero-Ruiz, 2012), con análisis más holísticos (Caffarel, Domínguez, Romano, 1989; De Aguilera, 1998; Jones, 1998; Martínez Nicolás, 2006, 2008; Martínez Nicolás, Saperas Lapiedra, 2011) o centrados en temáticas o líneas concretas (Almiron, Reig, 2007; Marcos Recio, Martínez Pestaña, Blasco López, 2012; Martínez Pestaña, 2011; Xifra, Castillo, 2006).

Estos trabajos apenas han tenido equivalencia a nivel autonómico, donde sólo en el caso de Cataluña ha habido una cierta tradición de análisis, constante aunque poco sistematizada, por el conjunto de la actividad científica (Berrio, 1997; Casasús i Guri, 2005; Gifreu i Pinsach, 1989; Jones, 2000; Moragas i Spà, Civil i Serra, Reguero i Jiménez, Sedó, 2007, 2009) o por aspectos concretos como los artículos científicos (Fernández-Quijada, 2011c, 2012; Masip, Fernández-Quijada, 2011) o las tesis doctorales (Jones, 1992; Jones, Baró i Queralt, 1997). Algún otro ejemplo aislado se da en Andalucía (Herrero Solana, Arboledas Márquez, 2011; Reig, García Orta, Moreno, Luque, 2000), el País Vasco (Landa Montenegro, 2004) o la Comunidad Valenciana (número monográfico de la revista Treballs de comunicació en 2007).

Los autores del presente artículo desconocen algún estudio equiparable a los anteriormente mencionados que se centre en la Comunidad de Madrid. Este hecho no deja de ser paradójico ya que el trabajo de Fernández-Quijada (2010) muestra que la Universidad Complutense se sitúa como la primera institución española en volumen de artículos publicados en revistas españolas de comunicación. Entre las diez primeras también figuran otras dos universidades públicas madrileñas, la Universidad Rey Juan Carlos y la Universidad Carlos III.

Las investigaciones de tipo bibliométrico centradas en la Comunidad de Madrid han sido del conjunto del sistema de ciencia y tecnología y, en línea con lo que sucede en comunicación, se muestra la centralidad de Madrid dentro del sistema español (Gómez et al., 2007, 2009; Morillo, De Filippo, 2009; Olmeda-Gómez et al., 2007) o 
se analizan sus pautas de colaboración (Olmeda-Gómez et al., 2006). Ante estos indicadores, parece justificado un trabajo que, como el presente, analice la producción científica en comunicación desarrollada en la Comunidad de Madrid. El objetivo es profundizar en las dinámicas de producción, publicación y colaboración de la investigación científica madrileña en comunicación a partir del análisis bibliométrico de la producción en revistas científicas del ámbito nacional. Junto a los libros, las revistas científicas son el principal instrumento de publicación de la actividad científica y así son valoradas por las agencias de evaluación. Además, la escasa internacionalización de la investigación española en comunicación hace que en este segmento se concentre el grueso de la producción. En términos prácticos, además, su estudio bibliométrico es mucho más accesible que el de los libros y, por ello, se han convertido en la principal fuente de datos de los estudios sobre ciencia y tecnología. En este caso, además, se decidió centrar el trabajo en los centros universitarios que, como muestra la mayoría de investigaciones reseñadas, suponen la práctica totalidad de centros en los que se realiza investigación publicada sobre la materia. Existen empresas u otras instituciones no-universitarias desde los que también se generan publicaciones pero son aportaciones aisladas que introducen ruido en los resultados, por lo que se decidió centrar el análisis en las instituciones que estructuralmente y de forma permanente generan ciencia sobre comunicación, las universidades.

Este objetivo general se desgranó en una serie de objetivos específicos de los que emergieron las tres preguntas de investigación que guiaron este trabajo. Así, un primer objetivo concreto era conocer la aportación de los diferentes centros universitarios al total de la producción científica en forma de artículos:

(P1) ¿Cómo se reparte la investigación en comunicación entre las universidades madrileñas?

Un segundo objetivo concreto era determinar cómo se repartía esta producción por revistas:

[P2] ¿Cuáles son las principales revistas en las que publican los investigadores madrileños?

Más allá de la desagregación de la producción por instituciones, la ciencia moderna considera la colaboración entre instituciones un elemento definitorio del dinamismo del sistema de ciencia y tecnología. Dada esta premisa, se quiso saber también cuánta colaboración había y cómo se estructuraba por universidades y revistas, de ahí que surgiera una tercera pregunta de investigación con dos subapartados, una sobre producción y otro sobre publicación:

(P3a) ¿Qué nivel y tipo de colaboración se da entre las universidades madrileñas en el ámbito de la comunicación?

(P3b) ¿Qué nivel y tipo de colaboración se da en la publicación científica madrileña en el ámbito de la comunicación? 


\section{MÉTODO}

A partir de los objetivos definidos inicialmente, este estudio bibliométrico se centró en dos tipos de indicadores: indicadores de producción y de colaboración. Para ello se tomaron como fuente de datos las principales revistas científicas de comunicación publicadas en España. En el estudio se trabajó con unidades agregadas, las instituciones de procedencia de los autores.El diseño metodológico se inició, como en otras investigaciones previas (Fernández-Quijada, 2011a), con la selección de la muestra de análisis. Con el objetivo de obtener una muestra representativa de las revistas españolas de comunicación, se optó por una triangulación de bases de datos. Concretamente, se seleccionaron como fuente las dos bases de datos de revistas españolas que ANECA (Agencia Nacional de Evaluación de la Calidad y Acreditación) suele utilizar como referencia al evaluar el ámbito de la comunicación: DICE (Difusión y calidad editorial de las revistas españolas de humanidades y ciencias sociales y jurídicas) y Latindex. A éstas se añadió IN-RECS (Índice de impacto de las revistas españolas de ciencias sociales) al ser la única que facilita datos sobre el impacto de las mismas mediante análisis de citas. De DICE se vaciaron las áreas de conocimiento de Periodismo y de Comunicación Audiovisual y Publicidad. De la sección de Comunicación de IN-RECS se obtuvo la lista de publicaciones que formaban su población. Finalmente, del ámbito de Ciencias de la Comunicación del Catálogo Latindex se obtuvieron las revistas editadas en España. Además, se hizo una búsqueda específica de las revistas aparecidas en la lista de DICE o IN-RECS que faltaban en esta nueva lista. De esta manera emergieron revistas adicionales clasificadas en áreas como Sociología, Bellas Artes, Ciencias de la Información, Lingüística o Cine.Se triangularon estas bases de datos para obtener el grupo de revistas nucleares de comunicación, aquellas que aparecían simultáneamente en estos tres índices. Al tratarse de un estudio longitudinal, cuyo período de análisis se inicia en 2007, en cada ola anual se añadieron revistas que cumplían con los parámetros de selección. Las fechas de cruce de las bases de datos fueron el 31 de marzo de 2009, y el 1 de julio de 2010 y 2011. De las nuevas revistas se contabilizaron sólo los datos de los artículos aparecidos el año anterior. Así, a partir de esta triangulación se obtuvo una lista de las que aparecían en las tres bases de datos y que, por tanto, pueden ser consideradas el núcleo de las revistas españolas de comunicación: Ámbitos, Anàlisi, Área abierta, CIC, Comunicación y sociedad, Comunicar, Doxa comunicación, Estudios sobre el mensaje periodístico, Historia y comunicación social, I/C, Questiones publicitarias, Revista latina de comunicación social, Trípodos y Zer para el período 2007-2008, a las que se deben añadir Icono 14 y Telos para 2009 y Archivos de la Filmoteca, Comunicación, Comunicación y hombre y Vivat academia para 2010.Una vez seleccionadas las revistas, se vaciaron los números correspondientes a los años 2007 a 2010 (a partir de 2009 para las revistas seleccionadas en 2010 y sólo 2010 en el caso de las revistas seleccionadas en 2011). De estos se escogieron para la muestra sólo los artículos firmados por autores pertenecientes a instituciones universitarias sitas en la Comunidad de Madrid, lo 
que supuso 390 artículos.De cada texto se analizaron diferentes variables como año de publicación, volumen de la revista, autores, título, idioma, institución de afiliación de los autores, país de procedencia de los autores y referencias. Para el objetivo del presente trabajo, no obstante, el análisis se centró en aspectos relacionados con la institución de afiliación de los autores. Siempre que estuvieran disponibles, se utilizaron las versiones electrónicas de los textos, dejando las versiones impresas sólo para los casos de embargo editorial o de problemas de acceso a los archivos digitales.Cabe señalar que se tuvo que decidir cómo medir la producción científica de las coautorías en los agregados, ya que se podían utilizar diversas opciones como la asignación exclusiva al primer autor, la fracción igual para cada autor, la fracción decreciente por orden de firma, etc. (Maltrás Barba, 2003). En esta investigación se optó por el criterio de asignación completa a instituciones; es decir, a cada coautor de diferente institución se le atribuyó todo el resultado, lo que provoca redundancia pero evita el oscurecimiento estadístico de las colaboraciones científicas resultante del resto de criterios posibles ${ }^{1}$.Para el análisis de la autoría se distinguió, en primer término, entre autoría individual y autoría múltiple. En este segundo caso, el detalle del análisis discernió entre trabajos elaborados por autores de una misma institución (sin colaboración), entre autores de la misma comunidad autónoma (colaboración regional), trabajos con autores pertenecientes a instituciones españolas más allá de la Comunidad de Madrid (colaboración nacional) y entre autores de centros madrileños e investigadores afiliados a instituciones de otros países (colaboración internacional). El análisis de colaboración, pues, se realiza agregadamente en el nivel institucional y no en el personal.Por otro lado, se decidió sumar la producción de los centros adscritos al total de su universidad matriz. Así, las contribuciones del Centro de Estudios Superiores Don Bosco, el Centro de Estudios Superiores Felipe II y el Centro Universitario Villanueva se contabilizaron como parte de la Universidad Complutense de Madrid, los artículos del Instituto de Estudios de la Democracia se contabilizaron como parte de la Universidad San Pablo CEU, las aportaciones del Centro de Gobierno Corporativo Bankinter/Iberdrola/IE/PwC se sumaron a las de IE University y las de la Escuela Superior de Gestión Comercial y Marketing (ESIC) a la Universidad Rey Juan Carlos.Adicionalmente, para complementar el análisis se aplicó una herramienta habitual en la investigación bibliométrica, el análisis de redes sociales (ARS). En este caso se emplearon Ucinet 6.382 (Borgatti y otros, 2002) y NetDraw 2.119 (Borgatti, 2002) para la representación gráfica de los sociogramas que muestran las relaciones. El primer sociograma (figura I) relaciona las instituciones madrileñas y las revistas en las que publican sus autores. Se trata, por tanto, de una matriz de modo 1. Las líneas son

\footnotetext{
${ }^{1}$ Esto significa que, por ejemplo, un artículo firmado por dos investigadores de la Universidad Complutense de Madrid se ha contabilizado como un artículo de esta universidad, mientras que si un autor del texto pertenecía a esta misma universidad y un segundo autor a la Universidad Rey Juan Carlos, se ha contabilizado un artículo para cada universidad.
} 
direccionales, expresando desde qué institución se publican en cada revista. Además, se trata de de líneas valoradas, que reflejan la intensidad de la relación a través de su grosor. El segundo sociograma (figura V), en cambio, se genera a partir de una matriz de modo 2 que expresa la colaboración en autoría de artículos entre las diferentes instituciones. El tamaño de los nodos indica el número total de colaboraciones, mientras que el grosor de las líneas indica el número de colaboraciones de cada relación diádica. Antes de analizar los resultados cabe matizar que no se deben leer como un análisis de productividad científica - un aspecto que debería tratarse junto a otros indicadores-, sino más bien como una exploración en el universo de las revistas que permita conocer mejor las dinámicas de producción y publicación que se generan en la comunidad científica madrileña dedicada a la investigación en comunicación.

\section{RESULTADOS}

Los resultados de esta investigación se presentan en dos bloques. Por un lado se analiza la distribución de las autorías de la producción científica en las diferentes universidades madrileñas y revistas académicas del ámbito de la comunicación. Por otro, se desglosan las dinámicas de colaboración de los centros y su relación con las diferentes publicaciones de la muestra.

\subsection{Distribución de autorías}

En el período comprendido entre 2007 y 2010, las universidades madrileñas participaron en 390 artículos publicados en las revistas de la muestra. El peso de los artículos en los que intervinieron investigadores de universidades madrileñas supone casi una tercera parte del total español, con una tendencia permanente de crecimiento, ya que entre 2007 y 2010 su participación aumenta en más de ocho puntos porcentuales (tabla I).

\section{TABLA I}

Artículos de instituciones madrileñas en revistas españolas de comunicación

\begin{tabular}{lccccc}
\hline & $\mathbf{2 0 0 7}$ & $\mathbf{2 0 0 8}$ & $\mathbf{2 0 0 9}$ & $\mathbf{2 0 1 0}$ & $\mathbf{2 0 0 7 - 1 0}$ \\
\hline Univ. Madrid & 56 & 83 & 123 & 128 & 390 \\
\hline Total España & 226 & 315 & 384 & 388 & 1.313 \\
\hline$\%$ univ. Madrid & $24,8 \%$ & $26,3 \%$ & $32 \%$ & $33 \%$ & $29,7 \%$ \\
\hline
\end{tabular}

Al desagregar estos datos por instituciones, se observa un fuerte liderazgo de la Universidad Complutense de Madrid, que participa en casi la mitad del total de la producción científica madrileña en comunicación (tabla II). A este liderazgo han contribuido también sus centros adscritos, que aportan 21 artículos, un $10,7 \%$ de la producción total de la Universidad Complutense. En un segundo plano permanecen la Universidad Rey Juan Carlos (19,7\%) y la Universidad Carlos III (14,6\%). En compa- 
ración, las universidades públicas son más activas que las privadas, que apenas firman el $12,1 \%$ del total de la producción, una séptima parte de la aportación de las universidades públicas. De hecho, algunas universidades públicas que no imparten estudios de comunicación, como la Universidad Nacional de Educación a Distancia o la Universidad Politécnica de Madrid, tienen más publicaciones que algunas universidades privadas con estos estudios. La excepción es la Universidad San Pablo CEU, que representa más de la mitad de la producción de los centros privados y se sitúa como cuarta institución con más artículos publicados. Su producción ha crecido con el paso de los años y, aunque en números totales la distancia entre la Universidad San Pablo CEU y la Universidad Carlos III es importante, en el 2010 sólo hubo un artículo de diferencia entre ambas.

TABLA II

Distribución de artículos por universidades

\begin{tabular}{lllllllllll}
\hline \multirow{2}{*}{ Institución } & $\mathbf{2 0 0 7}$ & \multicolumn{3}{c}{$\mathbf{2 0 0 8}$} & \multicolumn{2}{c}{$\mathbf{2 0 0 9}$} & \multicolumn{3}{c}{$\mathbf{2 0 1 0}$} & \multicolumn{2}{c}{$\mathbf{2 0 0 7 - 1 0}$} \\
\cline { 2 - 12 } & Art. & $\mathbf{\%}$ & Art. & $\mathbf{\%}$ & Art. & $\mathbf{\%}$ & Art. & \% & Art. & \% \\
\hline UCM & 37 & 63,8 & 40 & 47,6 & 62 & 46,3 & 58 & 42,6 & 197 & 47,8 \\
\hline URJC & 6 & 10,3 & 18 & 21,4 & 29 & 21,6 & 28 & 20,6 & 81 & 19,7 \\
\hline UC3M & 11 & 19 & 13 & 15,4 & 20 & 14,9 & 16 & 11,8 & 60 & 14,6 \\
\hline USPCEU & 3 & 5,2 & 3 & 3,6 & 6 & 4,5 & 15 & 11 & 27 & 6,6 \\
\hline UNED & & & 4 & 4,8 & 2 & 1,5 & 2 & 1,5 & 8 & 1,9 \\
\hline UPM & & & & & 3 & 2,2 & 4 & 2,9 & 7 & 1,7 \\
\hline UCJC & & & 2 & 2,4 & 2 & 1,5 & 2 & 1,5 & 6 & 1,5 \\
\hline UFV & & & 1 & 1,2 & & & 5 & 3,7 & 6 & 1,5 \\
\hline UAN & & & & & 2 & 1,5 & 3 & 2,2 & 5 & 1,2 \\
\hline CSIC & & & 1 & 1,2 & 3 & 2,2 & & & 4 & 1 \\
\hline UEM & 1 & 1,7 & 1 & 1,2 & 1 & 0,7 & & & 3 & 0,7 \\
\hline Otras & & & 1 & 1,2 & 4 & 2,8 & 3 & 2,2 & 8 & 1,9 \\
\hline Total & $\mathbf{5 8}$ & $\mathbf{1 0 0}$ & $\mathbf{8 4}$ & $\mathbf{1 0 0}$ & $\mathbf{1 3 4}$ & $\mathbf{1 0 0}$ & $\mathbf{1 3 6}$ & $\mathbf{1 0 0}$ & $\mathbf{4 1 2}$ & $\mathbf{1 0 0}$ \\
\hline
\end{tabular}

Nota: Los códigos de cada institución pueden comprobarse en el anexo I.

La distribución de la producción científica de los centros madrileños es, en números totales, fragmentada. Icono $14(11,8 \%)$, Estudios sobre el mensaje periodístico $(11 \%)$ y Telos $(9,7 \%)$ lideran la tabla de revistas que han publicado un mayor número de artículos firmados por autores de universidades madrileñas (tabla III). La única revista de la muestra que no ha publicado ningún artículo de universidades madrileñas es Comunicación. La razón es que se incorporó a la muestra en 2010, año en que sólo publicó cinco artículos. De las demás revistas, ninguna concentra un porcentaje elevado de artículos de autores madrileños. Entre la primera revista que más ha publicado (Icono 14) y la décima (Área abierta) apenas hay una diferencia de seis puntos porcentuales. Sin embargo, al analizar la media anual de artículos publicados las dife- 
rencias entre revistas se hacen más evidentes. Destacan las irrupciones de Icono $14 \mathrm{y}$ Telos, que a pesar de incorporarse en 2009 a la muestra, ya son dos de las tres revistas que más artículos de universidades madrileñas publican gracias a los 23 y 19 textos anuales de autores madrileños que incluyen de media en sus números, doblando a la siguiente revista, Estudios sobre el mensaje periodístico. Destaca también el aumento puntual de artículos de centros madrileños en revistas ajenas a la Comunidad de Madrid, como la Revista latina de comunicación social, editada por la Universidad de La Laguna o Comunicar, editada por el Grupo Comunicar. Ambas -Revista latina de comunicación social en 2009 y Comunicar en 2008- casi cuadruplican el número de textos de universidades madrileñas con respecto al año anterior.

TABLA III

Artículos publicados por autores de universidades madrileñas

\begin{tabular}{llllllll}
\hline Revista & $\mathbf{2 0 0 7}$ & $\mathbf{2 0 0 8}$ & $\mathbf{2 0 0 9}$ & $\mathbf{2 0 1 0}$ & $\mathbf{2 0 0 7}-\mathbf{1 0}$ & Media anual & $\mathbf{\%}$ \\
\hline II4 & & & 23 & 23 & 46 & 23 & 11,8 \\
\hline EMP & 9 & 13 & 11 & 10 & 43 & 10,8 & 11 \\
\hline Telos & & & 15 & 23 & 38 & 19 & 9,7 \\
\hline RLCS & 0 & 5 & 19 & 12 & 36 & 9 & 9,2 \\
\hline Comunicar & 4 & 15 & 8 & 4 & 31 & 7,8 & 8 \\
\hline CIC & 9 & 4 & 8 & 9 & 30 & 7,5 & 7,7 \\
\hline HCS & 9 & 7 & 6 & 6 & 28 & 7 & 7,2 \\
\hline Doxa & 5 & 7 & 10 & 4 & 26 & 6,5 & 6,7 \\
\hline Zer & 3 & 9 & 5 & 7 & 24 & 6 & 6,2 \\
\hline AA & 6 & 8 & 4 & 3 & 21 & 5,3 & 5,4 \\
\hline Ámbitos & 4 & 4 & 4 & 1 & 13 & 3,3 & 3,3 \\
\hline CyS & 2 & 4 & 3 & 3 & 12 & 3 & 3,1 \\
\hline Anàlisi & 2 & 4 & 3 & 0 & 9 & 2,3 & 2,3 \\
\hline CyH & & & & 9 & 9 & 9 & 2,3 \\
\hline I/C & 0 & 2 & 3 & 2 & 7 & 2,3 & 1,8 \\
\hline QP & 1 & 1 & 1 & 3 & 6 & 1,5 & 1,5 \\
\hline Vivat & & & & 4 & 4 & 4 & 1 \\
\hline AF & & & & 4 & 4 & 4 & 1 \\
\hline Trípodos & 2 & 0 & 0 & 1 & 3 & 0,8 & 0,8 \\
\hline Total & $\mathbf{5 6}$ & $\mathbf{8 3}$ & $\mathbf{1 2 3}$ & $\mathbf{1 2 8}$ & $\mathbf{3 9 0}$ & $\mathbf{9 7 , 5}$ & $\mathbf{1 0 0}$ \\
\hline
\end{tabular}

Nota: Los códigos de cada revista pueden comprobarse en el anexo II.

El análisis de redes sociales permite observar la centralidad de las diferentes universidades y revistas en el conjunto de la red (figura I). La proximidad de la posición de la Universidad Complutense, de la Universidad Rey Juan Carlos y de la Universi- 
dad Carlos III indica una similitud en los patrones de publicación de estas universidades, cuyos textos se distribuyen en revistas similares. El grosor de los enlaces entre revistas y universidades refleja la intensidad de la relación entre unas y otras a la hora de publicar. La posición periférica de Telos en esta red señala su capacidad de atracción de textos de centros ajenos a la comunicación, como la Universidad Politécnica de Madrid, que ha publicado todos sus artículos en esta revista. Otras universidades cuyas líneas principales de investigación no se relacionan directamente con la comunicación han encontrado en Telos una ventana de publicación específica, como IE University, la Escuela de Organización Industrial, la Universidad Autónoma de Madrid o la Universidad Nacional de Educación a Distancia.

La red también refleja las diferencias en las pautas de publicación de universidades con producciones similares. Por ejemplo, los seis artículos de la Universidad Francisco de Vitoria se han publicado en dos revistas (Comunicación y hombre y Comunicar), mientras que la Universidad Camilo José Cela, con la misma producción, ha publicado en cuatro revistas diferentes (Comunicación y hombre, Historia y comunicación social, Icono 14 y Zer

\section{FIGURA I}

Publicación de instituciones madrileñas en revistas españolas de comunicación

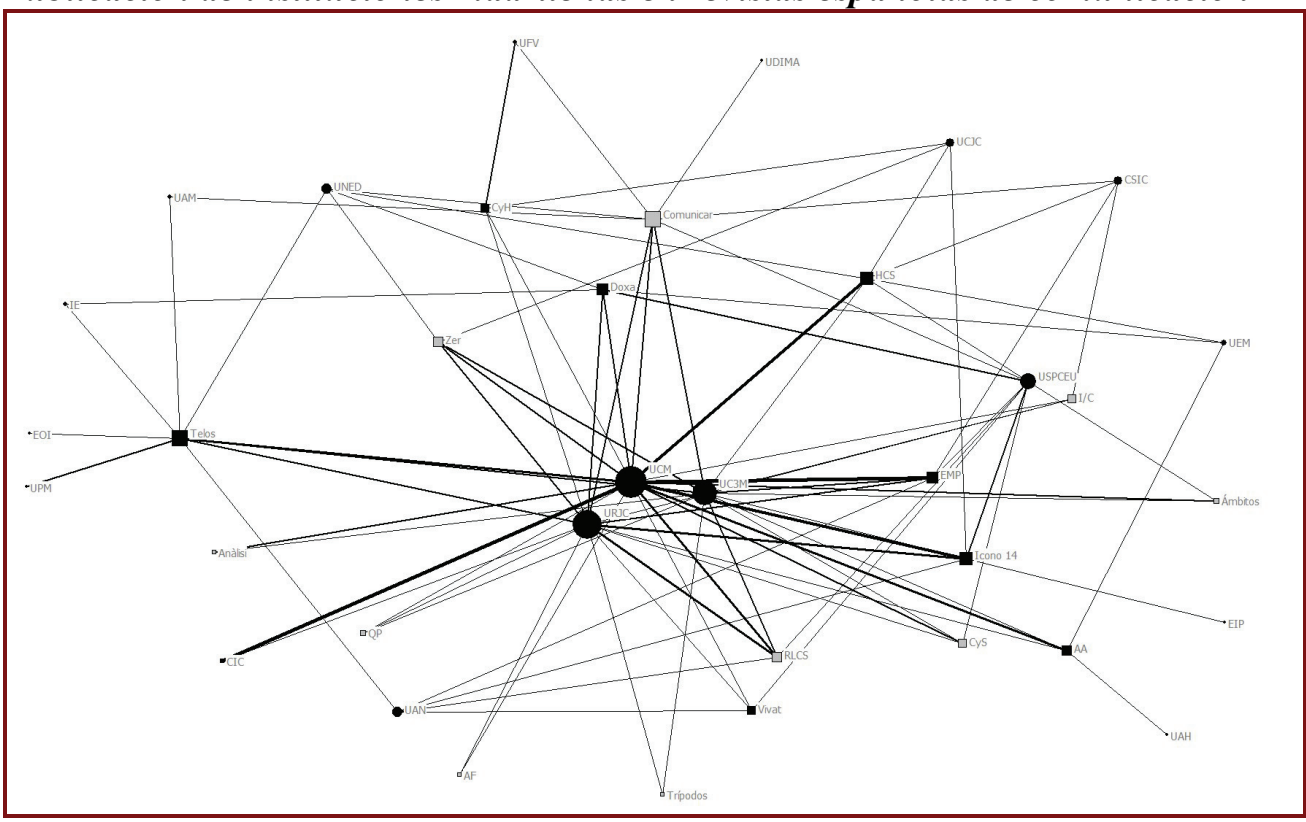

Nota: Las revistas editadas en la Comunidad de Madrid aparecen en negro, el resto en gris. 


\subsection{Dinámicas de colaboración}

De los 390 artículos en los que participaron investigadores de universidades madrileñas, 273 estaban firmados por un solo autor y 117 por dos o más. Aunque el porcentaje de autoría individual sigue siendo mayoritario, el número de coautorías muestra una tendencia creciente en los últimos cuatro años, doblándose entre 2007 y 2009, mientras que en 2010 retrocede ligeramente (figura II).

\section{FIGURA II}

\section{Tipo de autoría por número de autores}

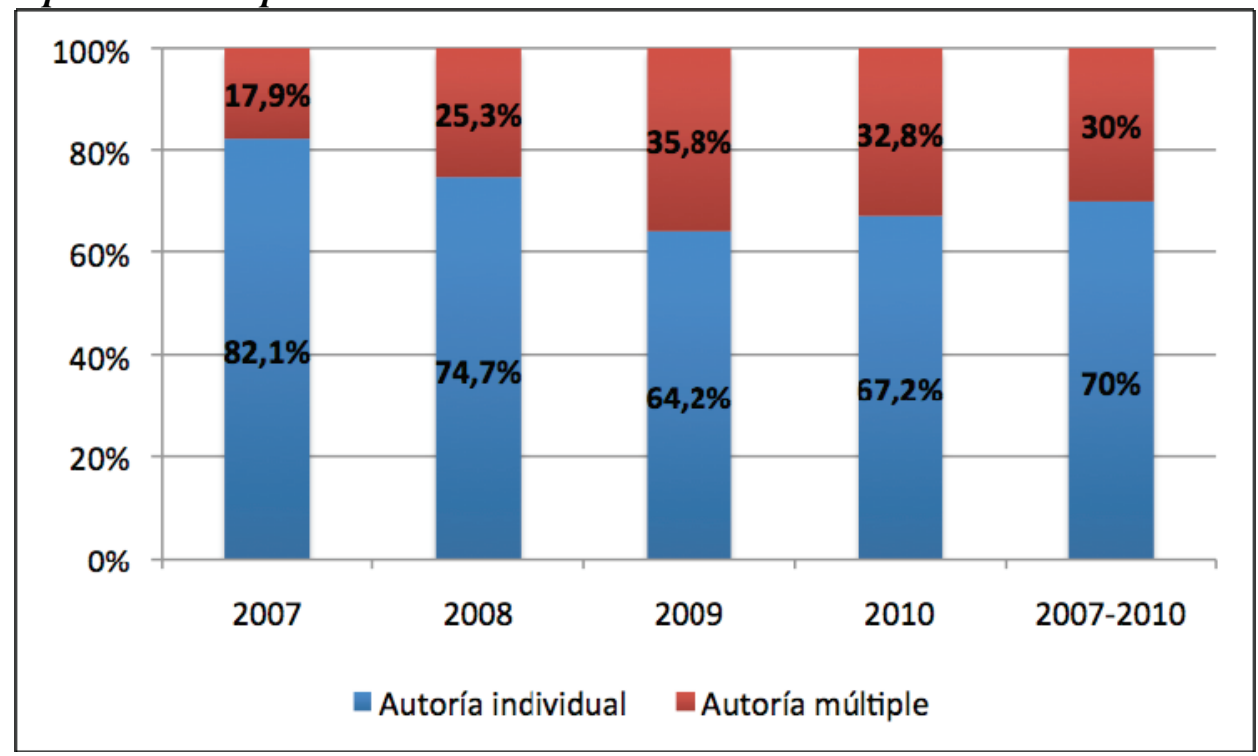

Este aumento ha tenido su impacto correspondiente sobre el índice de coautoría, que crece claramente durante el período de análisis (tabla IV). La Universidad San Pablo CEU presenta el índice más alto de coautorías, con una media de dos autores por artículo. La Universidad Rey Juan Carlos $(1,74)$ también se sitúa por encima de la media, mientras que la Complutense $(1,42)$ y la Universidad Carlos III $(1,33)$ mantienen índices más bajos.

TABLA IV

\begin{tabular}{|l|l|l|l|l|l|l|l|l|l|l|}
\hline & \multicolumn{9}{|l|}{$\mathbf{2 0 0 7}$} & \multicolumn{3}{|l|}{$\mathbf{2 0 0 8}$} \\
\hline Universidad & $\mathbf{1}$ & $\mathbf{2}$ & $\mathbf{3}$ & $\mathbf{+ 4}$ & IC & $\mathbf{1}$ & $\mathbf{2}$ & $\mathbf{3}$ & $\mathbf{+ 4}$ & IC \\
\hline UCM & 30 & 5 & 2 & & 1,24 & 31 & 5 & 1 & 3 & 1,45 \\
\hline
\end{tabular}




\begin{tabular}{|l|l|l|l|l|l|l|l|l|l|l|}
\hline URJC & 5 & 1 & & & 1,16 & 12 & 4 & & 2 & 1,61 \\
\hline UC3M & 10 & 1 & & & 1,10 & 8 & 5 & & & 1,39 \\
\hline USPCEU & & 1 & 2 & & 2,67 & 2 & & & 1 & 2,33 \\
\hline Otras & 1 & & & & 1 & 9 & 1 & & & 1,10 \\
\hline Total & $\mathbf{4 6}$ & $\mathbf{8}$ & $\mathbf{4}$ & $\mathbf{0}$ & $\mathbf{1 , 2 8}$ & $\mathbf{6 2}$ & $\mathbf{1 5}$ & $\mathbf{1}$ & $\mathbf{6}$ & $\mathbf{1 , 4 8}$ \\
\hline
\end{tabular}

\begin{tabular}{|l|l|l|l|l|l|l|l|l|l|l|}
\hline & \multicolumn{9}{|l}{2009} & \multicolumn{1}{l|}{2010} \\
\hline $\begin{array}{c}\text { Uni- } \\
\text { versidad }\end{array}$ & $\mathbf{1}$ & $\mathbf{2}$ & $\mathbf{1}$ & $\mathbf{2}$ & $\mathbf{1}$ & $\mathbf{2}$ & $\mathbf{1}$ & $\mathbf{2}$ & $\mathbf{1}$ & IC \\
\hline UCM & 42 & 12 & 42 & 12 & 42 & 12 & 42 & 12 & 42 & 1,45 \\
\hline URJC & 11 & 11 & 11 & 11 & 11 & 11 & 11 & 11 & 11 & 1,61 \\
\hline UC3M & 15 & 3 & 15 & 3 & 15 & 3 & 15 & 3 & 15 & 1,39 \\
\hline USPCEU & 2 & 4 & 2 & 4 & 2 & 4 & 2 & 4 & 2 & 2,33 \\
\hline Otras & 9 & 6 & 9 & 6 & 9 & 6 & 9 & 6 & 9 & 1,10 \\
\hline Total & $\mathbf{7 9}$ & $\mathbf{3 6}$ & $\mathbf{7 9}$ & $\mathbf{3 6}$ & $\mathbf{7 9}$ & $\mathbf{3 6}$ & $\mathbf{7 9}$ & $\mathbf{3 6}$ & $\mathbf{7 9}$ & $\mathbf{1 , 4 8}$ \\
\hline
\end{tabular}

La autoría individual es la opción más común en los artículos registrados en la muestra. En línea con los resultados del índice de coautoría, esta tendencia se acentúa en las universidades con un bajo índice de coautoría, como la Universidad Complutense (un $72,6 \%$ de sus artículos son de autoría individual) y la Universidad Carlos III $(73,3 \%)$ mientras que las cifras disminuyen en el caso de la Universidad Rey Juan Carlos $(51,2 \%)$ y la Universidad San Pablo CEU (40,7\%).

Sin embargo, el análisis de las coautorías apunta ciertas diferencias en las dinámicas de colaboración (tabla V). En 2007 y 2008 la colaboración entre investigadores de una misma institución suponía más del 50\% de colaboraciones. En 2009 el número de autorías múltiples duplica el del año anterior y junto a este aumento también se aprecia un crecimiento de colaboraciones regionales, nacionales e internacionales. La inclusión en la muestra de Icono 14 y Telos ha contribuido a esta situación, pues ambas concentran, junto con Revista latina de comunicación social, el 52,3\% de las colaboraciones de los últimos dos años. 
TABLA V

Tipología de autoría múltiple

\begin{tabular}{lllllllllll}
\hline Colaboraciones & \multicolumn{2}{l}{$\mathbf{2 0 0 7}$} & \multicolumn{2}{l}{$\mathbf{2 0 0 8}$} & \multicolumn{2}{l}{$\mathbf{2 0 0 9}$} & \multicolumn{2}{c}{$\mathbf{2 0 1 0}$} & \multicolumn{2}{c}{$\mathbf{2 0 0 7 - 2 0 1 0}$} \\
\cline { 2 - 12 } & $\mathbf{n}$ & $\mathbf{\%}$ & $\mathbf{n}$ & $\mathbf{\%}$ & $\mathbf{N}$ & $\mathbf{\%}$ & $\mathbf{n}$ & $\mathbf{\%}$ & $\mathbf{n}$ & $\mathbf{\%}$ \\
\hline $\begin{array}{l}\text { Sin colabora- } \\
\text { ción }\end{array}$ & 6 & 46,2 & 15 & 62,5 & 24 & 38,1 & 21 & 36,2 & 66 & 41,8 \\
\hline Regional & 4 & 30,8 & 4 & 16,7 & 21 & 33,3 & 17 & 29,3 & 46 & 29,1 \\
\hline Nacional & 2 & 15,4 & 4 & 16,7 & 13 & 20,6 & 17 & 29,3 & 36 & 22,8 \\
\hline Internacional & 1 & 7,7 & 1 & 4,1 & 5 & 7,9 & 3 & 5,2 & 10 & 6,3 \\
\hline Total & $\mathbf{1 3}$ & $\mathbf{1 0 0}$ & $\mathbf{2 4}$ & $\mathbf{1 0 0}$ & $\mathbf{6 3}$ & $\mathbf{1 0 0}$ & $\mathbf{5 8}$ & $\mathbf{1 0 0}$ & $\mathbf{1 5 8}$ & $\mathbf{1 0 0}$ \\
\hline
\end{tabular}

Nota: los diferentes tipos de colaboraciones pueden aparecer simultáneamente en un mismo artículo. En los casos en los que no constaba institución y/o país para alguno de los coautores, se han analizado sólo los datos disponibles.

El tipo de colaboración más habitual en este período se da entre autores de una misma institución, aunque cada vez con menor diferencia. Los patrones de este tipo de artículos repiten los datos totales de producción. La institución madrileña que en estos cuatro años ha intervenido en un mayor número de colaboraciones ha sido la Universidad Complutense de Madrid (tabla VI). De las 65 coautorías en las que ha participado, seis provienen de sus centros adscritos (tres colaboraciones nacionales y tres autorías múltiples entre investigadores de la Universidad Complutense de Madrid). Tras la Universidad Complutense, la Universidad Rey Juan Carlos es la universidad con más colaboraciones. En tercer lugar aparece la Universidad San Pablo CEU, que supera a la Universidad Carlos III gracias a sus colaboraciones regionales e internacionales. En el caso de la Universidad San Pablo CEU, el trabajo de sus centros adscritos también es relevante, pues representa uno de los siete artículos con colaboración dentro de la institución, uno de los siete con instituciones regionales y uno de los tres firmados con autores extranjeros. Si sólo se han encontrado cuatro instituciones que han firmado artículos con este tipo de colaboración, en el caso de la colaboración regional el número de universidades crece hasta nueve y siete en la colaboración nacional. La Universidad Complutense de Madrid lidera todas las clasificaciones de colaboración excepto la colaboración nacional, que comparte con la Universidad Rey Juan Carlos. Ambas suman el 65,3\% de las colaboraciones regionales y el $66,7 \%$ de los artículos de instituciones madrileñas firmados en colaboración nacional. 
TABLA VI

Tipología de autoría múltiple por universidades

\begin{tabular}{llllllllllll}
\hline Sin colaboración & Regional & \multicolumn{3}{c}{ Nacional } & \multicolumn{3}{c}{ Internacional } \\
\hline $\begin{array}{l}\text { Institu- } \\
\text { ción }\end{array}$ & $\mathbf{n}$ & $\mathbf{\%}$ & $\begin{array}{l}\text { Institu- } \\
\text { ción }\end{array}$ & $\mathbf{n}$ & $\mathbf{\%}$ & $\begin{array}{l}\text { Institu- } \\
\text { ción }\end{array}$ & $\mathbf{n}$ & $\mathbf{\%}$ & $\begin{array}{l}\text { Institu- } \\
\text { ción }\end{array}$ & $\mathbf{n}$ & $\mathbf{\%}$ \\
\hline UCM & 3 & 48, & UCM & 1 & 37 & UCM & 1 & 33, & UCM & 4 & 40 \\
& 2 & 5 & & 7 & & & 2 & 3 & & & \\
\hline URJC & 1 & 27, & URJC & 1 & 28, & URJC & 1 & 33, & USPCE & 3 & 30 \\
& 8 & 3 & & 3 & 3 & & 2 & 3 & U & & \\
\hline UC3M & 9 & 13, & USPCE & 7 & 15, & UC3M & 5 & 13, & UCJC & 1 & 10 \\
& & 6 & U & & 2 & & & 9 & & & \\
\hline USPCE & 7 & 10, & UAN & 2 & 4,3 & USPCE & 4 & 11, & UNED & 1 & 10 \\
U & 6 & & & & U & & 1 & & & \\
\hline & & & UCJC & 2 & 4,3 & CSIC & 1 & 2,8 & URJC & 1 & 10 \\
\hline & & UC3M & 2 & 4,3 & UCJC & 1 & 2,8 & & & \\
\hline & & EIP & 1 & 2,2 & UNED & 1 & 2,8 & & & \\
\hline & & UDIMA & 1 & 2,2 & & & & & & \\
\hline & & UFV & 1 & 2,2 & & & & & \\
\hline Total & $\mathbf{6}$ & $\mathbf{1 0 0}$ & Total & $\mathbf{4}$ & $\mathbf{1 0 0}$ & Total & $\mathbf{3}$ & $\mathbf{1 0 0}$ & Total & $\mathbf{1}$ & $\mathbf{1 0}$ \\
& $\mathbf{6}$ & & $\mathbf{6}$ & & & $\mathbf{6}$ & & & $\mathbf{0}$ & $\mathbf{0}$ \\
\hline
\end{tabular}

La figura IV presenta los patrones de colaboración de las universidades madrileñas. El reducido número de colaboraciones de algunos centros (CSIC, Escuela Internacional de Protocolo, Universidad Antonio de Nebrija, Universidad Camilo José Cela, Universidad a Distancia de Madrid, Universidad Francisco de Vitoria, Universidad Nacional de Educación a Distancia) no permite extraer conclusiones sobre sus dinámicas de colaboración. Sin embargo, las analogías y diferencias de las cuatro universidades más productivas quedan representados. La Universidad Carlos III, que apenas colabora a nivel regional o no tiene colaboraciones internacionales, es la tercera institución con más coautorías nacionales y la única cuya mayoría de colaboraciones se establece entre sus propios investigadores. Las universidades Complutense, Rey Juan Carlos y San Pablo CEU muestran pautas más desglosadas. La autoría múltiple sin colaboración es la más habitual, pero no es mayoritaria. Las colaboraciones regionales, nacionales e internacionales tienen un peso cada vez mayor en estos centros. En el caso de la Universidad San Pablo CEU, dos de cada tres artículos elaborados en colaboración están firmados con autores de instituciones ajenas. 


\section{FIGURA IV}

\section{Distribución de autoría múltiple por universidades}

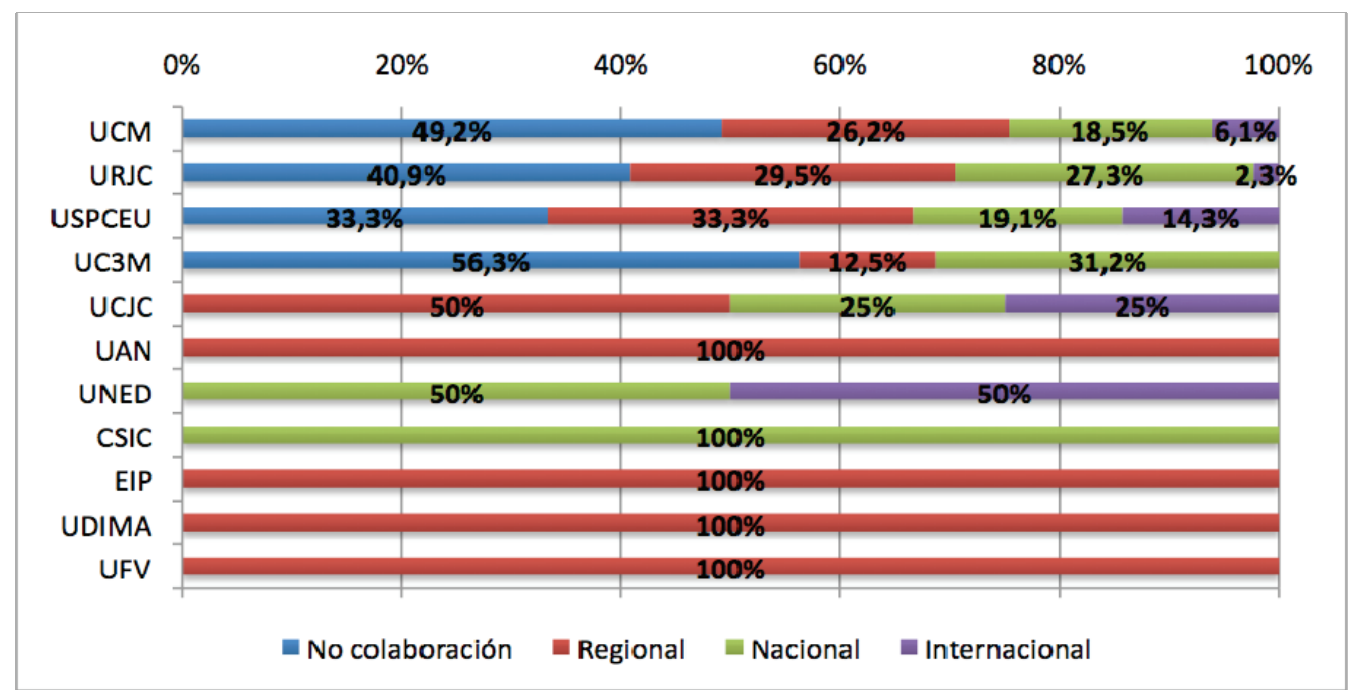

La red de colaboraciones entre instituciones se puede observar en el ARS de la figura 5. El cálculo de rango (degree) muestra la posición relevante de la Universidad Complutense (19), seguida por la Universidad Rey Juan Carlos (14). La primera privada, la Universidad San Pablo CEU (9) aparece por delante la Universidad Carlos III (8). El triángulo principal de colaboración se establece entre la Universidad Complutense, la Universidad Rey Juan Carlos y la Universidad San Pablo CEU. De hecho, esta universidad privada colabora más con las dos primeras que la Universidad Carlos III, que apenas tiene dos colaboraciones con otras universidades madrileñas: una con la Universidad Complutense y una con la Universidad Rey Juan Carlos. Además, la Universidad San Pablo CEU sirve como nexo de colaboración con la red madrileña a cinco instituciones externas a ella, mientras que la Universidad Carlos III lo hace con dos. Una institución externa a la Comunidad de Madrid, la Universidad de Valladolid, aparece más centrada en la red que algunas regionales gracias a que ha colaborado con las cuatro principales universidades. La Universidad Nacional de Educación a Distancia está desconectada de la red debido a su nula colaboración con instituciones de Madrid. Por último, se observa que la colaboración internacional está limitada a trabajos puntuales, de ahí la situación periférica de los distintos centros extranjeros. 


\section{FIGURA V}

\section{Red de colaboración de las instituciones madrileñas}

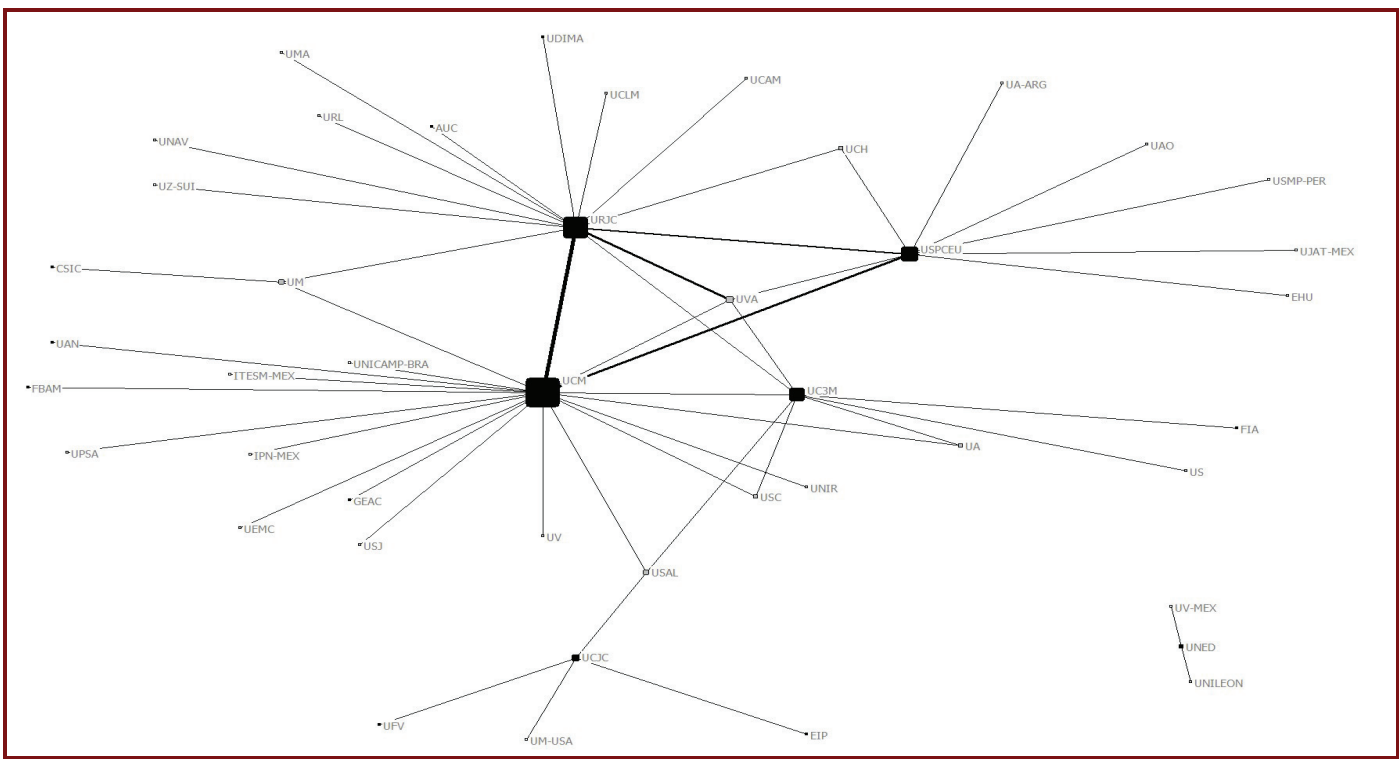

Nota: Las instituciones de la Comunidad de Madrid aparecen marcadas en negro, el resto de españolas en gris y las internacionales en blanco.

De las veinte revistas de la muestra, diecisiete han publicado algún artículo de autoría múltiple en el que interviene al menos una universidad madrileña. La autoría individual, sin embargo, sigue siendo el método mayoritario en todas las revistas. Sólo Ámbitos, Comunicación y sociedad y Revista latina de comunicación social presentan un porcentaje de artículos en coautoría igual o superior al de autoría individual. Además de Comunicación, que no ha publicado ningún artículo de investigadores madrileños en este período, ni Trípodos ni Anàlisi han publicado artículos en coautoría de universidades madrileñas. También han publicado esencialmente artículos firmados por un solo investigador Área abierta (95,2\% de sus artículos), Cuadernos de Información y Comunicación (93,3\%) e Historia y Comunicación Social $(85,7 \%)$. Los artículos escritos en colaboración se han publicado principalmente en Revista latina de comunicación social (17,4\%), Icono 14 (16,7\%), Doxa $(9,8 \%)$, Comunicar $(9,1 \%)$ y Estudios del mensaje periodístico (tabla VII). Solo se han registrado ocho revistas que hayan publicado todos los tipos de colaboraciones (Ámbitos, Comunicar, Comunicación y sociedad, Doxa, Estudios sobre el mensaje periodístico, Icono 14, Revista latina de comunicación social y Zer). 
TABLA VII

Tipología de autoría múltiple por revistas

\begin{tabular}{|c|c|c|c|c|c|c|c|c|c|c|c|}
\hline \multicolumn{3}{|c|}{ Sin colaboración } & \multicolumn{2}{|c|}{ Regional } & \multicolumn{4}{|c|}{ Nacional } & \multicolumn{3}{|c|}{ Internacional } \\
\hline Revista & $\bar{n}$ & $\%$ & Revista & $\bar{n}$ & $\%$ & Revista & $\mathbf{n}$ & $\%$ & Revista & $\bar{n}$ & $\%$ \\
\hline RLCS & $\begin{array}{l}1 \\
3\end{array}$ & 20 & $\mathrm{I} 14$ & 6 & 25 & RLCS & 6 & $\begin{array}{l}18, \\
2\end{array}$ & $\begin{array}{l}\text { Ámbi- } \\
\text { tos }\end{array}$ & 2 & 20 \\
\hline $\mathrm{I} 14$ & $\begin{array}{l}1 \\
0\end{array}$ & $\begin{array}{l}15 \\
4\end{array}$ & $\begin{array}{l}\text { Comu- } \\
\text { nicar }\end{array}$ & 3 & $\begin{array}{l}12 \\
5 \\
\end{array}$ & I14 & 5 & $\begin{array}{l}15, \\
2\end{array}$ & $\begin{array}{l}\text { Comu- } \\
\text { nicar }\end{array}$ & 1 & 10 \\
\hline Doxa & 6 & 9,2 & Doxa & 3 & $\begin{array}{l}12, \\
5\end{array}$ & Zer & 5 & $\begin{array}{l}15, \\
2\end{array}$ & $\mathrm{CyS}$ & 1 & 10 \\
\hline $\begin{array}{l}\text { Ámbi- } \\
\text { tos }\end{array}$ & 5 & 7,7 & RLCS & 3 & $\begin{array}{l}12, \\
5\end{array}$ & EMP & 4 & $\begin{array}{l}12, \\
1\end{array}$ & Doxa & 1 & 10 \\
\hline CyS & 5 & 7,7 & HCS & 2 & 8,3 & $\begin{array}{l}\text { Comu- } \\
\text { nicar }\end{array}$ & 3 & 9,1 & EMP & 1 & 10 \\
\hline $\begin{array}{l}\text { Comu- } \\
\text { nicar }\end{array}$ & 5 & 7,7 & $\begin{array}{l}\text { Ámbi- } \\
\text { tos }\end{array}$ & 1 & 4,2 & Doxa & 3 & 9,1 & I14 & 1 & 10 \\
\hline EMP & 5 & 7,7 & $\mathrm{CyH}$ & 1 & 4,2 & Telos & 2 & 6,1 & RLCS & 1 & 10 \\
\hline Telos & 5 & 7,7 & $\mathrm{CyS}$ & 1 & 4,2 & $\begin{array}{l}\text { Ámbi- } \\
\text { tos }\end{array}$ & 1 & 3 & Telos & 1 & 10 \\
\hline $\mathrm{HCS}$ & 3 & 4,6 & EMP & 1 & 4,2 & $\mathrm{AF}$ & 1 & 3 & Zer & 1 & 10 \\
\hline $\mathrm{CIC}$ & 2 & 3 & QP & 1 & 4,2 & $\mathrm{CyS}$ & 1 & 3 & & & \\
\hline QP & 2 & 3 & Vivat & 1 & 4,2 & $\mathrm{I} / \mathrm{C}$ & 1 & 3 & & & \\
\hline $\mathrm{AA}$ & 1 & 1,5 & Zer & 1 & 4,2 & QP & 1 & 3 & & & \\
\hline $\mathrm{CyH}$ & 1 & 1,5 & & & & & & & & & \\
\hline $\mathrm{I} / \mathrm{C}$ & 1 & 1,5 & & & & & & & & & \\
\hline Zer & 1 & 1,5 & & & & & & & & & \\
\hline Total & $\begin{array}{l}6 \\
5\end{array}$ & $\overline{100}$ & Total & $\begin{array}{l}2 \\
4\end{array}$ & 100 & Total & $\begin{array}{l}3 \\
3\end{array}$ & 100 & Total & $\begin{array}{l}1 \\
0\end{array}$ & $\begin{array}{l}10 \\
0\end{array}$ \\
\hline
\end{tabular}

La colaboración más habitual es entre autores de una misma universidad. Se han identificado casi tantas de este tipo como de todas las demás sumadas. Revista latina de comunicación social e Icono 14 son las que más artículos de autoría múltiple sin colaboración han publicado. La revista en la que se encuentra un mayor número de artículos de colaboración regional es Icono 14. Telos, que ha publicado 38 artículos de instituciones madrileñas en los últimos dos años, no ha incluido en sus volúmenes ninguna colaboración regional. En colaboraciones nacionales destacan Revista latina de comunicación social, Icono 14, Zer y Estudios sobre el Mensaje Periodístico. Zer ha publicado más colaboraciones nacionales de investigadores madrileños que sumando los demás tipos. A nivel internacional el panorama es diferente. Se han encontrado diez artículos firmados entre investigadores de centros madrileños y de institu- 
ciones extranjeras en nueve revistas diferentes. Sólo Ámbitos ha publicado más de un artículo con colaboración internacional, pero nunca en años consecutivos.

\section{DISCUSIÓN Y CONCLUSIONES}

El análisis de la producción científica de las instituciones madrileñas en el ámbito de la comunicación permite extraer algunas conclusiones relacionadas con el peso de la Comunidad de Madrid en la producción total española, el peso de las diferentes universidades, las dinámicas de colaboración entre ellas y las principales revistas en las que se publican los artículos elaborados por sus investigadores. La primera pregunta de investigación se interesaba por el reparto de la producción entre universidades madrileñas. Los resultados presentan un panorama oligopólico, con cuatro universidades (Universidad Complutense de Madrid, Universidad Rey Juan Carlos, Universidad Carlos III y Universidad San Pablo CEU) que concentran un 88,7\% de toda la producción. A la luz de los datos, el predominio de la Universidad Complutense de Madrid resulta innegable. Ciertamente, a la tradición por ser pionera de los estudios de comunicación en España une el mayor tamaño de entre las universidades españolas y, por tanto, la mayor masa de investigadores. Aunque más que dobla a la siguiente universidad en producción científica, la Universidad Rey Juan Carlos, su peso porcentual disminuye un tercio a lo largo del período. Además, los centros adscritos a la Universidad Complutense han tenido una importancia relevante y creciente en estos años. Si se interpretara su producción como independiente, estos centros adscritos se situarían como la quinta institución con mayor producción de la Comunidad de Madrid. A pesar de que los datos son bastante estables a lo largo del período, sí que se observan algunos que llaman la atención y para los que no se halla una explicación. Por un lado, la producción de la Universidad Rey Juan Carlos para 2007, seis artículos, se triplica y quintuplica en los dos años posteriores. En términos porcentuales es la mitad de su aportación en el resto de años, que se mantiene altamente estable, lo que parece indicar que la anomalía reside en la baja producción para 2007. De hecho, en 2007 la producción de la Universidad Rey Juan Carlos es casi la mitad que la de la Universidad Carlos III, mientras que a partir de 2008 ésta queda por debajo claramente, casi en la mitad en 2010. Como en el caso de la Complutense, el peso de la Universidad Carlos III en la producción científica madrileña no ha dejado de caer en este período. Sin embargo, el descenso de ésta ha sido más acusado pues ha perdido, en comparación, una mayor cuota de producción. Al otro lado se encuentra la Universidad San Pablo CEU, cuya trayectoria ha sido de constante crecimiento. En cuatro años ha quintuplicado su producción de artículos y ha duplicado su peso porcentual en la investigación madrileña. En todo caso, el papel de las universidades privadas continúa siendo muy limitado en el ámbito de la investigación, ya que no llega ni al $15 \%$ de toda la producción de la Comunidad de Madrid. La segunda pregunta de investigación ponía el foco en las revistas científicas de comunicación. Des- 
de 2007 las universidades madrileñas han tenido un peso cada vez más importante en la producción nacional de artículos científicos de comunicación publicados en revistas españolas. La media en este período se sitúa cerca del 30\%, pero en 2010 han estado involucradas en uno de cada tres artículos nacionales. Esta tendencia se podría explicar por la creciente presencia en la muestra de revistas editadas en la propia Comunidad de Madrid (5 de 14 en 2009, 7 de 16 en 2010 y 9 de 20 en 2010). Es necesario puntualizar que las dinámicas de publicación y la selección de revistas por parte de los investigadores puede variar notablemente y dependiendo del ámbito de estudio, la especialización temática o la proyección internacional de las investigaciones es posible que algunos autores hayan optado por enviar sus trabajos a revistas extranjeras o nacionales ajenas a la muestra. En el caso de la Universidad Complutense de Madrid, decana de los estudios de comunicación en España, sus revistas son referencia a la hora de publicar. Seis de las veinte revistas fuente utilizadas en la muestra están editadas en esta universidad, lo que indica el notable trabajo editorial que realiza la institución. Icono 14, Estudios sobre el mensaje periodístico, Cuadernos de información y comunicación, Historia y comunicación social, Área abierta y Vivat academia han publicado en este período el $44 \%$ de los artículos en los que han participado universidades madrileñas. La influencia de las revistas de la Universidad Complutense de Madrid se refleja también en las investigaciones propias. Las seis revistas editadas por sus departamentos y grupos de investigación publican el 57,3\% de los artículos firmados por sus investigadores, lo que refleja el localismo de esta producción a la hora de ser difundida y cierta endogamia de algunas de estas revistas. Esta circunstancia también se observa en algunas universidades privadas que editan revistas de comunicación. Los investigadores de la Universidad San Pablo CEU han publicado en los últimos cuatro años un $34,3 \%$ de sus artículos en Doxa comunicación, la revista que edita su universidad. De forma similar, cinco de los seis artículos firmados por investigadores de la Universidad Francisco de Vitoria han sido publicados en Comunicación y hombre, la revista editada por esta universidad. Atendiendo a la tercera pregunta de investigación, se ha verificado por un lado la colaboración entre las diferentes universidades y por otro las dinámicas de publicación de los artículos en coautoría en las revistas de la muestra. En los últimos cuatro años la colaboración más habitual es la que se establece entre investigadores de un mismo centro, pero la tendencia es decreciente a favor de colaboraciones nacionales y regionales. Sin embargo, el crecimiento en número de coautorías no se refleja en un aumento de las colaboraciones internacionales, que dependen de vínculos puntuales de investigadores locales con alguna institución extranjera, lo que demuestra su fragilidad. Alternativamente, si estos vínculos existen es posible que se estén vehiculando en publicaciones del ámbito internacional, más allá del objeto de estudio del presente trabajo. Los datos desagregados por instituciones vuelven a expresar la fuerte posición de la Universidad Complutense de Madrid en casi todas las formas de autoría múltiple. Las principales diferencias en los patrones de colaboración aparecen en las demás instituciones. Algunas como la Universidad Rey Juan Carlos concentran sus colaboraciones princi- 
palmente en el territorio nacional, ya sea entre investigadores de la propia universidad, autores de otros centros madrileños o investigadores de otros centros españoles. En la Universidad Carlos III los autores colaboran principalmente entre sí y con otras instituciones españolas pero apenas firman con otros investigadores madrileños o extranjeros. De hecho, sólo se ha encontrado un artículo en el que un investigador de la Carlos III firme con otros autores de centros de la Comunidad de Madrid. Sin duda llama la atención la escasa colaboración entre la Universidad Carlos III y las demás universidades madrileñas. Esta universidad actúa prácticamente como una isla dentro de la Comunidad de Madrid en términos de colaboración. La Universidad San Pablo CEU muestra un comportamiento inverso al de la Carlos III, pues colabora más con instituciones madrileñas e internacionales, además de firmar la mayoría de sus colaboraciones con investigadores de la propia universidad. Otro dato singular es el que destaca a la Universidad de Valladolid, que no pertenece a la Comunidad de Madrid pero aparece en una posición más centrada en la red de colaboraciones de las universidades madrileñas, por encima de muchas privadas o de la propia Carlos III.La observación de los patrones de publicación de los artículos en colaboración también arroja datos interesantes. La publicación de textos de autoría individual es la regla. Las excepciones se encuentran en tres revistas editadas fuera de Madrid. Ámbitos, Comunicación y sociedad y Revista latina de comunicación social han publicado tantos o más artículos de instituciones madrileñas en colaboración que de autoría individual. A pesar de que en números totales las revistas editadas en Madrid en general y en la Complutense en particular son las que atraen más textos de investigadores madrileños, los artículos en colaboración también aparecen publicados en revistas ajenas a la comunidad, como Revista latina de comunicación social, que es la que más colaboración madrileña publica, Comunicar, que aparece entre las revistas que más colaboración regional madrileña incluye, Zer, que es la segunda publicación que atrae más colaboraciones nacionales o Ámbitos, que es la revista que ha publicado más artículos de centros madrileños con colaboración internacional. Este trabajo abre una línea de análisis de la producción científica en el ámbito de la comunicación de las universidades de la Comunidad de Madrid. El análisis, centrado en una amplia muestra de las revistas académicas más relevantes de nuestro país, dibuja un primer estado de la cuestión que tiene aplicaciones prácticas tanto sobre las políticas de investigación de las universidades como sobre las políticas editoriales de las revistas. Igualmente, marca unos primeros puntos de referencia para futuras investigaciones que se interesen por las dinámicas de trabajo de las instituciones madrileñas que investigan en el área de la comunicación. Para completar el análisis de este objeto de estudio se podrían verificar en posteriores trabajos otras fuentes de datos como revistas internacionales, libros o presentaciones en congresos que complementen el examen de su estado de la cuestión. 


\section{BIBLIOGRAFÍA}

ALMIRON, Núria; Reig, Ramón. «The communications research in Spain: the Political Economy epistemological approach», American Communication Journal, 2007, 9/2 (http://acjournal.org/journa1/2007/Summer/5CommunicationsResearchinSpain.pdf) Consulta: $18-5-12$.

BALADRÓN-PAZOS, Antonio J.; Correyero-Ruiz, Beatriz. «Futuro de las revistas científicas de comunicación en España», El profesional de la información, 2012, $21 / 1,34-42$.

BERRIO, Jordi (Dir.): Un segle de recerca sobre comunicació a Catalunya. Estudi crític dels principals àmbits d'investigació de la comunicació de massa. Bellaterra: Universitat Autònoma de Barcelona, 1997.

BORGATTI, Stephen P.: NetDraw: Graph Visualization Software. Harvard: Analytic Technologies, 2002.

BORGATTI, Stephen P.; Everett, Martin G.; Freeman, Linton C.: Ucinet 6 for Windows: Software for Social Network Analysis. Harvard: Analytic Technologies, 2002.

CAFFAREL, Carmen; DOMÍNGUEZ, Milagros;ROMANO, Vicente. «El estado de la investigación de comunicación en España (1978-1987)», C.in.co, 1989, 3, 4557.

CASASÚS I GUIRU, Josep Maria (Coord.): Reports de la recerca a Catalunya. Comunicació i informació 1996-2002. Barcelona: Institut d'Estudis Catalans, 2005.

CASTILLO, Antonio; CARRETÓN, M Marmen. «Investigación en Comunicación. Estudio bibliométrico de las Revistas de Comunicación en España», Comunicación y sociedad, 2010, XXIII/2, 289-327.

DE AGUILERA, Miguel. «La investigación sobre comunicación en España: una visión panorámica», Comunicación y cultura, 1998, 4, 5-11.

FERNÁNDEZ-QUIJADA, David. «El perfil de las revistas españolas de comunicación (2007-2008)», Revista española de documentación científica, 2010, 33/4, 553-581.

FERNÁNDEZ-QUIJADA, David. De los investigadores a las redes: una aproximación tipológica a la autoría en las revistas españolas de comunicación. En Piñuel Raigada, José Luis; Lozano, Carlos y García Jiménez, Antonio (Eds.): Investigar la comunicación en España. Fuenlabrada, Asociación Española de Investigación de la Comunicación/Universidad Rey Juan Carlos, 2011a [CD-ROM].

FERNÁNDEZ-QUIJADA, David. «Appraising internationality in Spanish communication journals», Journal of Scholarly Publishing, 2011b, 43/1, 90-109.

FERNÁNDEZ-QUIJADA, David. «Aproximación bibliométrica a la investigación catalana en comunicación (2007-2009)», BiD: textos universitaris de biblioteconomia $i$ documentació, 2011c, 26. (http://www.ub.edu/bid/26/fernandez2.htm) Consulta: 18-5-12. 
FERNÁNDEZ-QUIJADA, David. «Dues dècades de comunicació científica: anàlisi bibliomètrica de les revistes de la Societat Catalana de Comunicació (19892009)», Comunicació. Revista de recerca i d'anàlisi, 2012, 29/1, 125-151.

GIFREU I PINSACH, Josep. «La investigació de la comunicació a Catalunya: assaig de periodització», Anàlisi, 1989, 12, 9-65.

GIMÉNEZ-TOLEDO, Elea: La opinión de los expertos sobre las revistas españolas de comunicación y otros indicadores de calidad. En Piñuel Raigada, José Luis; Lozano Ascensio, Carlos y García Jiménez, Antonio (Eds.): Investigar la comunicación en España. Fuenlabrada, Asociación Española de Investigación de la Comunicación/Universidad Rey Juan Carlos, 2011 [CD-ROM].

GIMÉNEZ-TOLEDO, Elea;ALCAIN PARTEARROYO, María Dolores. «Estudio de las revistas españolas de periodismo», Comunicación y sociedad, 2006, XIX/2, 107-131.

GIMÉNEZ-TOLEDO, Elea; TEJADA-ARTIGAS Carlos. «Valoración de editoriales especializadas en Comunicación, Biblioteconomía y Documentación: encuesta a profesores e investigadores», El profesional de la información, 2012, 21/1, 50-62.

GÓMEZ, Isabel; Bordons, María; Morillo, Fernanda; Moreno, Luz; Aparicio, Javier; Candelario, Antonia; González-Albo, Borja; Herrero, María: Indicadores de Producción Científica de la Comunidad de Madrid (2004-2008). Madrid: Consejo Superior de Investigaciones Científicas, 2009.

GÓMEZ, Isabel; Fernández, María Teresa.; Bordons, María; Morillo, Fernanda; Aparicio, Javier; Candelario, Antonia; De Filippo, Daniela; González-Albo, Borja; Herrero, María; Moreno, Luz: Indicadores de Producción Científica y Tecnológica de la Comunidad de Madrid (PIPCYT). Madrid: Dirección General de Universidades e Investigación. Consejería de Educación. Comunidad de Madrid, 2007.

HERRERO SOLANA, Víctor; Arboledas Márquez, Luis. «Una aproximación temática a la producción andaluza en comunicación: análisis a partir de las tesis doctorales de las Universidades de Sevilla y de Málaga», Hipertext.net, 2011, 9 (http://www.upf.edu/hipertextnet/numero-9/produccion-andaluzacomunicacion.html) Consulta: 18-5-12.

JONES, Daniel E. «Dues dècades de recerca en comunicació: Ciències de la Informació ha fet possibles 70 tesis doctorals i 85 de llicenciatura», Capçalera, 1992, 32, 21-23.

JONES, Daniel E. «Investigación sobre comunicación en España. Evolución y perspectivas», Zer, 1998, 5, 13-51.

JONES, Daniel E. «Investigació sobre comunicació a Catalunya als anys noranta», Treballs de comunicació, 2000, 13-14, 41-55.

JONES, Daniel E.: La comunicación en el escaparate. En Díaz-Nosty, Bernardo (Dir.): Tendencias'07. Medios de comunicación: el escenario iberoamericano. Barcelona, Madrid, Ariel, Fundación Telefónica, 2007, pp. 395-408. 
JONES, Daniel E.; Baró i Queralt, Jaume. «Tesis doctorals i treballs de recerca universitaris sobre comunicació als Països Catalans, 1954-1996», Anàlisi, 1997, 20, 157-187.

LANDA MONTENEGRO, Carmelo. «Recopilación de tesis doctorales sobre Comunicación en las Universidades del País Vasco (1996-2000)», Mediatika, 2004, 10, 267-295.

LÓPEZ RABADÁN, Pablo; Vicente-Mariño, Miguel: Métodos y técnicas de investigación dominantes en las Revistas científicas españolas sobre comunicación (2000-2009). En Piñuel Raigada, José Luis; Lozano Ascensio, Carlos y García Jiménez, Antonio (Eds.): Investigar la comunicación en España. Fuenlabrada, Asociación Española de Investigación de la Comunicación/Universidad Rey Juan Carlos, 2011 [CD-ROM].

MALTRÁS BARBAS, Bruno: Los indicadores bibliométricos. Fundamentos y aplicación al análisis de la ciencia. Gijón: Trea, 2003.

MARCOS RECIO, Juan Carlos; Martínez Pestaña, María Jesús; Blasco López, María Francisca. «Producción y dirección de tesis doctorales sobre publicidad en la universidad española (1971-2010)», Revista española de documentación científica, 2012, 35/3, 433-452.

MARTÍNEZ NICOLÁS, Manuel. «Masa (en situación) crítica. La investigación sobre periodismo en España: comunidad científica e intereses de conocimiento», Anàlisi, 2006, 33, 135-170.

MARTÍNEZ NICOLÁS, Manuel: La investigación sobre comunicación en España. Evolución histórica y retos actuales. En: Martínez Nicolás, Manuel (Coord.): Para investigar la comunicación. Propuestas teórico-metodológicas. Madrid, Tecnos, 2008, pp. 13-52.

MARTÍNEZ NICOLÁS, Manuel; Saperas Lapiedra, Enric. «La investigación sobre Comunicación en España (1998-2007). Análisis de los artículos publicados en revistas científicas», Revista latina de comunicación social, 2011, 66, 101-129 (http://www.revistalatinacs.org/11/art/926_Vicalvaro/05_Nicolas.html) Consulta: 18-5-2012.

MARTÍNEZ PESTAÑA, María Jesús. «La investigación universitaria en Publicidad: producción y temática de las tesis doctorales (1971-2001)», Documentación de las ciencias de la información, 2011, 34, 223-239.

MASIP, Pere. «Efecto Aneca: producción española en comunicación en el Social science citation index», Anuario ThinkEPI, 2011a, 5, 206-210.

MASIP, Pere: Los efectos del efecto Aneca: análisis de la producción Española en comunicación en el Social Science Citation Index (1999-2009). En: Piñuel Raigada, José Luis; Lozano Ascensio, Carlos y García Jiménez, Antonio (Eds.): Investigar la comunicación en España. Fuenlabrada, Asociación Española de Investigación de la Comunicación/Universidad Rey Juan Carlos, 2011b [CD-ROM].

MASIP, Pere; Fernández-Quijada, David. «Mapping Communication Research in Catalonia: a comparative analysis of publication patterns in scholarly journals 
(2007-09)», Catalan Journal of Communication \& Cultural Studies, 2011, 3/1, 95108.

MORAGAS I SPÀ, Miquel de; Civil i Serra, Marta; Reguero i Jiménez, Núria; Sedó, Ramon G.: La recerca i els estudis sobre comunicació a Catalunya. Estat de la qüestió (2005 i 2006). En Moragas i Spà, Miquel de; Fernández Alonso, Isabel; Blasco Gil, José Joaquín; Guimerà i Orts, Josep Àngel; Corbella Cordomí, Joan; Civil i Serra, Marta y Gibert i Fortuny, Oriol (Eds.): Informe de la comunicació a Catalunya 2005-2006. Bellaterra, Edicions UAB, 2007, pp. 317-347.

MORAGAS I SPÀ, Miquel de; Civil i Serra, Marta; Reguero i Jiménez, Núria; Sedó, Ramon G.: La recerca i els estudis sobre comunicació a Catalunya. Estat de la qüestió (2007 i 2008). En Moragas i Spà, Miquel de; Fernández Alonso, Isabel; Almiron, Núria; Blasco Gil, José Joaquín; Corbella Cordomí, Joan; Civil i Serra, Marta y Gibert i Fortuny, Oriol (Eds.): Informe de la comunicación a Catalunya 2007-2008. Barcelona, Generalitat de Catalunya, 2009, pp. 355-400.

MORILLO, Fernanda; De Filippo, Daniela. «Descentralización de la actividad científica. El papel determinante de las regiones centrales: el caso de Madrid», Revista española de documentación científica, 2009, 32/3, 29-50.

OLMEDA-GÓMEZ, Carlos; Ortiz-Repiso Jiménez, Virginia; Aragón González, Inés; Ovalle-Perandones, María Antonia y Periane-Rodríguez, Antonio: Indicadores cientificos de Madrid. ISI, Web of Science (1990-2003). Madrid: Comunidad de Madrid, 2007. (http://eprints.rclis.org/bitstream/10760/10238/1/iccm.pdf) Consulta: $15-9-12$.

Olmeda-Gómez, Carlos; Periane-Rodríguez, Antonio; Ovalle-Perandones, María Antonia; Ortiz-Repiso Jiménez, Virginia y Aragón González, Inés: Representación de la colaboración autonómica de la Comunidad de Madrid mediante patrones de coautoría (1995-2003). En Guerrero Bote, VP (Ed.): Proceedings I International Conference on Multidisplinary Information Science. Badajoz, Instituto Abierto del Conocimiento, 2006, pp. 625-629.

REIG, Ramón; García Orta, María José; Moreno, Lourdes; Luque, Javier. «Investigación sobre comunicación en Sevilla. Once años de la Facultad de Ciencias de la Información (1989-2000)», Ámbitos, 2000, 5, 259-311.

XIFRA, Jordi; Castillo, Antonio. «Forty years of doctoral public relations research in Spain: a quantitative study of dissertation contribution to theory development», Public Relations Review, 2006, 32/3, 302-308.

\section{ANEXOS}

\section{Anexo I: Códigos institucionales}

AUC $=$ Asociación de Usuarios de la Comunicación

$\mathrm{CSIC}=$ Consejo Superior de Investigaciones Científicas

$\mathrm{EHU}=\mathrm{U}$. del País Vasco/Euskal Herriko Unibertsitatea

EIP $=$ Escuela Internacional de Protocolo 
$\mathrm{EOI}=$ Escuela de Organización Industrial

FBAM = Fundación Banco de Alimentos de Madrid

FIA $=$ Fundación Infancia y Aprendizaje

GEAC $=$ Gabinete de Estudios en Acciones Comunicativas

IPN-MEX = Instituto Politécnico Nacional (México)

ITESM-MEX = Instituto Tecnológico y de Estudios Superiores de Monterrey

$\mathrm{UA}=\mathrm{U} \cdot \mathrm{d}$ 'Alacant

$\mathrm{UA}-\mathrm{ARG}=\mathrm{U}$. Austral (Argentina)

$\mathrm{UAH}=\mathrm{U}$. Alcalá de Henares

$\mathrm{UAM}=\mathrm{U}$. Autónoma de Madrid

$\mathrm{UAN}=\mathrm{U}$. Antonio de Nebrija

$\mathrm{UAO}=\mathrm{U}$. Abat Oliba CEU

$\mathrm{UC} 3 \mathrm{M}=\mathrm{U}$. Carlos III de Madrid

$\mathrm{UCAM}=\mathrm{U}$. Católica de Murcia

$\mathrm{UCH}=\mathrm{U}$. Cardenal Herrera-CEU

$\mathrm{UCJC}=\mathrm{U}$. Camilo José Cela

$\mathrm{UCLM}=\mathrm{U}$. de Castilla la Mancha

$\mathrm{UCM}=\mathrm{U}$. Complutense de Madrid

UDIMA $=$ U. a Distancia de Madrid

$\mathrm{UEM}=\mathrm{U}$. Europea de Madrid

$\mathrm{UEMC}=\mathrm{U}$. Europea Miguel de Cervantes

$\mathrm{UFV}=\mathrm{U}$. Francisco de Vitoria

UJAT-MEX = U. Juárez Autónoma de Tabasco (México)

$\mathrm{UM}=\mathrm{U}$. de Murcia

UM-USA = U. Maryland (EE.UU.)

$\mathrm{UMA}=\mathrm{U}$. de Málaga

$\mathrm{UNAV}=\mathrm{U}$. de Navarra

$\mathrm{UNED}=\mathrm{U}$. Nacional de Educación a Distancia

UNICAMP-BRA = U. Estadual de Campinas (Brasil)

UNILEON $=$ U. de León

$\mathrm{UNIR}=\mathrm{U}$. Internacional de La Rioja

$\mathrm{UPM}=$ U. Politécnica de Madrid

UPSA $=$ U. Pontifica de Salamanca

$\mathrm{URJC}=\mathrm{U}$. Rey Juan Carlos

$\mathrm{URL}=\mathrm{U}$. Ramon Llull

$\mathrm{US}=\mathrm{U}$. de Sevilla

$\mathrm{USAL}=\mathrm{U}$. de Salamanca

$\mathrm{USC}=\mathrm{U}$. de Santiago de Compostela

$\mathrm{USJ}=\mathrm{U}$. San Jorge

USMP-PER $=$ U. San Martín de Porres (Perú)

$\mathrm{USPCEU}=\mathrm{U}$. San Pablo-CEU

$\mathrm{UV}=\mathrm{U}$. de València 
UV-MEX $=$ U. Veracruzana (México)

$\mathrm{UVA}=\mathrm{U}$. de Valladolid

$\mathrm{UZ}-\mathrm{SUI}=\mathrm{U}$. Zurich (Suiza)

Anexo II: Códigos de revistas

$\mathrm{AA}=$ Área abierta

$\mathrm{AF}=$ Archivos de la Filmoteca

Ámbitos = Ámbitos. Revista internacional de comunicación

Anàlisi = Anàlisi: quaderns de comunicació $i$ cultura

$\mathrm{CIC}=$ Cuadernos de información y comunicación

Comunicar $=$ Comunicar. Revista cientifica iberoamericana de comunicación y educación

$\mathrm{CyH}=$ Comunicación y hombre

$\mathrm{CyS}=$ Comunicación y sociedad

Doxa $=$ Doxa comunicación

$\mathrm{EMP}=$ Estudios sobre el mensaje periodístico

$\mathrm{HCS}=$ Historia y comunicación social

$\mathrm{I} / \mathrm{C}=I / C$. Información y comunicación

$\mathrm{I} 14$ = Icono14. Revista de comunicación y tecnologías emergentes

$\mathrm{QP}=$ Questiones publicitarias

RLCS = Revista latina de comunicación social

Telos = Telos. Cuadernos de comunicación, tecnología y sociedad

Vivat $=$ Vivat Academia

Zer $=$ Zer. Revista de estudios de comunicación 